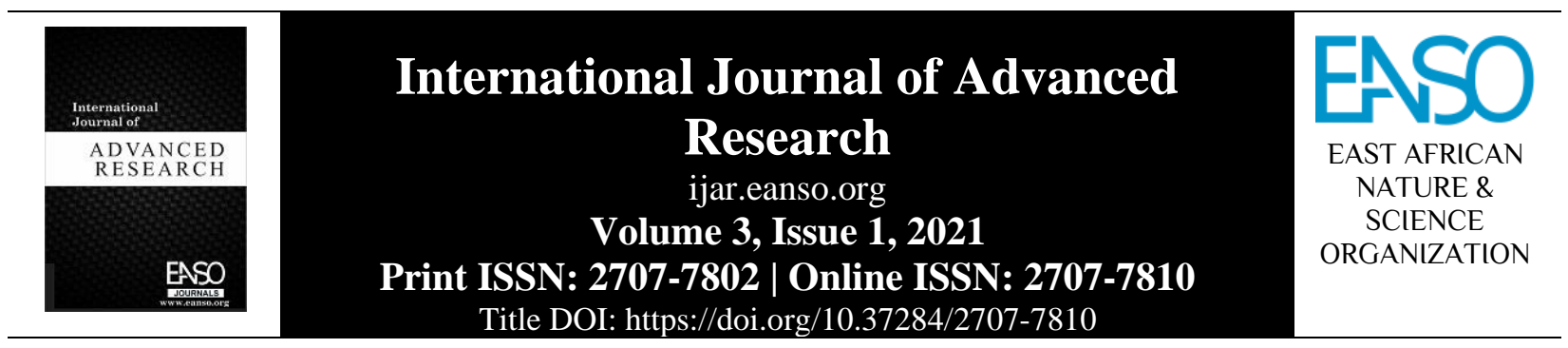

Original Article

\title{
Exploration of Human Figure Drawings Using Charcoal Pencil - Analysis of Post-Graduate Drawings by Zephania Lukamba
}

\author{
Dr. Kamau Wango, $P h D^{1 *}$ \\ ${ }^{1}$ Department of Fine Art and Design, Kenyatta University, P. O. Box 43844 - 00100, Nairobi, Kenya. \\ * ORCID: https://orcid.org/0000-0002-0185-1355; Author for Correspondence email: kamauwango@gmail.com.
}

Article DOI: https://doi.org/10.37284/ijar.3.1.306

Publication Date:

24 March 2021

Keywords:

Figure Drawing,

Proportions,

Value,

Illumination,

Gestures,

Movement.

\section{ABSTRACT}

Human figure drawing is undertaken and ultimately used for a number of purposes. Artists use it to continually sharpen their skills in order to apply it in the execution of their work in artistic disciplines that pertain to self-expression. Students and other groups as well as individuals embark on human figure drawing in order to acquire and horn their skills for purposes of artistic development that is then applied ultimately to their respective artistic endeavours. However, the drawing and acquisition of skills is a process and people render their human figure drawings to different levels of success and finesse at any given stage. In this process, one draws human figures using certain prescribed guidelines. It is expected that as one works within this process, particularly in a formal learning environment like studio-based work, following these guidelines become essential and helpful in attaining a proportional and accurate human figure drawing. In analysing the featured work executed on toned paper, this paper seeks to determine the extent to which the artist applies the basic tenets of human figure drawing and whether the drawings themselves attain this threshold. The analytical framework includes the depiction of correct proportions, the study of gestures, the suggestion of movement and application of value. Within the development of personal style, the artist specifically explores the effect of charcoal pencil on toned paper as his medium of choice. His methodology includes the application of a variety of tones and the use of focused illumination upon pertinent areas in the drawings to create deliberate effects that highlight the drawings, enhance gestures, suggest movement and add dynamism to the drawings. The drawings include photograph referenced male and female figures as well as separate studies of hands and feet 
International Journal of Advanced Research, Volume 3, Issue 1, 2021

Article DOI: https://doi.org/10.37284/ijar.3.1.306

\section{APA CITATION}

Wango, K. (2021). Exploration of Human Figure Drawings Using Charcoal Pencil - Analysis of Post-Graduate Drawings by Zephania Lukamba. International Journal of Advanced Research, 3(1), 55-71. https://doi.org/10.37284/ijar.3.1.306

\section{CHICAGO CITATION}

Wango, Kamau. 2021. "Exploration of Human Figure Drawings Using Charcoal Pencil - Analysis of Post-Graduate Drawings by Zephania Lukamba". International Journal of Advanced Research 3 (1), 55-71. https://doi.org/10.37284/ijar.3.1.306.

\section{HARVARD CITATION}

Wango, K. (2021) "Exploration of Human Figure Drawings Using Charcoal Pencil - Analysis of Post-Graduate Drawings by Zephania Lukamba”, International Journal of Advanced Research, 3(1), pp. 55-71. doi: 10.37284/ijar.3.1.306.

\section{IEEE CITATION}

K. Wango, "Exploration of Human Figure Drawings Using Charcoal Pencil - Analysis of Post-Graduate Drawings by Zephania Lukamba", IJAR, vol. 3, no. 1, pp. 55-71, Mar. 2021.

\section{MLA CITATION}

Wango, Kamau. "Exploration of Human Figure Drawings Using Charcoal Pencil - Analysis of Post-Graduate Drawings by Zephania Lukamba". International Journal of Advanced Research, Vol. 3, no. 1, Mar. 2021, pp. 55-71, doi:10.37284/ijar.3.1.306.

\section{INTRODUCTION}

Human figure drawing, whether derived from direct observation of a specific model (life drawing) or from transient forms that are unaware that they are the subject of study or from other references such as photographs, is a captivating and essential artistic undertaking. The human body itself is a complex form that is fascinating to observe and study from its entirety to that of specific parts such as hands, feet, head and face. Human figure drawing necessitates the generation and study of gestures that emanate from action-oriented poses. Gestures then become an important avenue for the expression of a whole retinue of human feelings, emotions, and moods as they manifest themselves.

When artists use gestures in their human figure depictions in their paintings and sculptures, these gestures underscore the gist of expression that the individual artist wishes to propagate. Robinson (2007) observes that "representational paintings and sculptures, though usually immobile, can express emotions by depicting people who are expressing their emotions in facial expression, posture, gestures, and action tendencies." When artists use figure drawings as compositional elements in their work, be it in their paintings or sculptures, they are in essence using the gestural value of these forms in propagating certain desired expressions. Facial expressions are critical in augmenting gestural expressions and that is why the study of holistic figure drawing is important. However, because of the need to zero-in on facial expression, artists find it uniquely useful to study the face on its own, just as they isolate hands and feet. But ultimately, all these become part of the complete whole. One important aspect of this gestural value is the suggestion of movement in drawings, a key tenet of human figure drawing.

Human figure drawing is done from two perspectives; the study of the female figure as well as that of the male figure. Both elicit different inspirations and perhaps by virtue of their nature, anatomical composition, perceptions, historical associations and symbolism, draw different descriptions of artistic expression, expressiveness, passion, and drama.

Human figure drawing is fundamental because it will always remain central in the creation of art as well as forming the foundation of the artist's own creativity, education and inclination towards selfexpression. When drawing a human figure, one embarks on multiple aspects of artistic visual value such as perspective, proportions, gestures, and movement that contribute to all-round skill development. Apart from studying the normal anatomical principles, one also studies weight, balance, and tension within the body's functionality.

The exploration of different types of media and techniques as well as the continuous engagement with drawing is essential in the acquisition and development of skills that raise the level and ability towards expression. Artists approach human figure 
drawing according to the objectives at hand. Some may be looking for quick gesture drawings, or more realistic renditions, or specific techniques that they wish to apply. Some often use human figure drawings as an avenue for their own subtle expression. The artist's work, as featured in this paper, depicts great interest in the intricacies of the human figure. The artist takes time to illustrate poses, movement, proportions and study of parts of the human body. He uses value to add life and excitement to his figure studies, making them more convincing and relatable. In some drawings, he uses sharp contrasts to illuminate certain parts of the drawings to create a much more engaging form than its photographic reference.

\section{EXPRESSIVENESS OF THE HUMAN FIGURE}

Probably, even before delving into the human figure, a question that often manifests itself in related artistic discourse is what is the essence of drawing itself in this regard and what quality does it bring to the human figure. Hutter (2020) perhaps provides a comprehensive response to this when he observes that "formally, drawing offers the widest possible scope for the expression of artistic intentions. Bodies, space, depth, substantiality, and even motion can be made visible through drawing." When drawing the human figure, an artist fundamentally deals with the body and its composition, motion as constituted in the joints, the formulation of gestures and suggestion of movement as well as use of perspective. So there exists a visual correlation between the essence of drawing and the integral dynamics of human figure drawing. The spontaneity of drawing provides the artist with the opportunity to explore the human figure, for instance, in a way that underscores personal expression. Hutter notes "furthermore, because of the immediacy of its statement, drawing expresses the draftsperson's personality spontaneously in the flow of the line; it is, in fact, the most personal of all artistic statements." Human figure drawing by its own definition has the ability to create interesting narratives through the human form it embodies, mainly because of its associative value and the fact that it is easily relatable.

\section{Expressiveness of the Female Figure}

Even though the use of the female body in art has evolved over time, its usefulness and appeal in the realm of the artist's mind has continued to be an intriguing subject of discussion, often drawing emotive passions. This has generally culminated in two distinct schools of thought each of whose opinion, even though focusing on the same subject, is the complete opposite of the other.

It cannot be denied that among artists, the female body has been a subject of study and fascination for many centuries where it has been the source of artistic exploration, creativity and in some circumstances, obsession. Walter (2019) observes that 'for centuries the female body has been an obsession in art. Many artists have used the female form as a muse or medium, around which they create and explore.' Having a glimpse of the representation of women and the female body through the ages. Pelissier (2018) observes, for instance, that starting from the middle ages "women often tend to be represented in a very polarised way as either good or bad, saint or sinner, idealised or erotic, respectable or fallen." Delving into the use of the female nude by a male-dominated cohort of artists, Pelissier further observes that "the female nude has often been sexualised or objectified to some degree, as well as idealised. The female nudes tend to conform to the ideal of beauty of their time."

One of the major thematic tenets of fascination with the female body is the concept of nakedness. Subsequently, although the art world keeps evolving, it seems unlikely that this fascination will diminish or radically shift, mainly because the female body shall not cease to be born and artists shall not cease to exist. Walter (2019) notes "the fascination with the female form in art often corresponds with the naked body." This incessant quest to explore women in the nude has not come without its controversy, particularly the feeling that the female body has been more often than not viewed merely from a sexual perspective. Errington (2015) observes that "the female body in an artistic form has always had the capability to arouse and ignite sexual passions." Pelissier (2018) notes that, "unfortunately, there are also countless representations of objectified women conforming to a unique stereotype of what beauty is thought to be." 
There is, however, the school of thought which encompasses the feelings of many artists that see the female body as bearing the ability to transcend mere sexual representation or individual gratification. The female body instead becomes a non-sexualised symbol of other universal themes, particularly its symbolism pertaining to nature and the environment. In this regard, Errington (2015) observes that"

The female body has the ability to transcend beyond sexual representation, depending upon an artist's intentions. While there have been and still are artists who use the female body to symbolise sexuality, there have been other artists able to portray the complete opposite.

Irrespective of the side of the aisle that an artist may subscribe to, it is clear that the female body, by its own nature and creation, will remain a major subject of inspiration, fascination, passion and obsession among artists in the realm of human figure representation. It is also clear that while the arguments against sexualisation, objectification and even idealisation of the female body are sound, it shall remain difficult, perhaps unattainable, to remove some certain perceptions and tendencies from the minds of all artists because many postulates that these form an integral part of their fascination and motivation and are not erasable. It can be argued that when the female nude body is seen and interpreted as a non-sexualised symbol of other universal themes, then it bears its unique expressiveness. When the female nude is observed within the confines of sexualisation and objectification and becomes merely a subject of the artist's own gratification and personal expression, then it loses its expressiveness and broader appeal to propagate other universal themes.

\section{Expressiveness of the Male Figure}

The male figure is perhaps seen as less controversial than the female figure in terms of visual scrutiny. The female form is often subject to a wider spectrum of interrogation in terms of symbolism, sexual objectification and perception as a passive object of desire as well as stereotyped cultural representation. The male figure is often depicted culturally as a symbol of masculinity and strength mainly because its musculoskeletal structure is physically modelled as such and this definition suits certain social/cultural narratives. Some have argued that the male figure too bears its own aesthetical beauty in terms of its physical structure and flow while others have insisted that the emanation of 'beauty' is confined only to the female form.

\section{Use of the Nude}

Nude models are used in life drawing so as to capture the holistic human figure as it is naturally modelled as well as to internalise its essence and its beauty. When the human body is seen as an essential expressive tool for the artist, and is articulated in a non-prejudicial manner, then the unadorned human form becomes uniquely useful. Artists approach this emotive matter from the point of view of the complexity and beauty of the human form as a piece of creation and how it manifests itself in the natural world.

\section{ANALYTICAL FRAMEWORK}

In analysing the drawings in this paper, it is important to take cognisance of the following points

\section{Proportions}

Both the male and female figure should depict correct body proportions from head to toe. Proportions are always based on the concept of the human anatomy, which encompasses the musculoskeletal configuration of the parts of the body and their functions.

\section{Gestural Poses}

One cannot study the human figure devoid of gestural poses since gestures denote movement and action and hence demonstrate body functionality. The study of the interrelations between parts of the body is done by examining endless gestural poses that underscore the spontaneity of the human form that make it exciting to interrogate.

\section{Movement}

The depiction of visual movement in a figure drawing is important in adding life and dynamism to the figure since the human form itself is not static and dull; it is fluid and dynamic. This is closely related to the mechanics of joints. The idea is to 
create an illusion or sense of movement within the stationary pose, giving it rhythm and dynamism. Winslow (2008) observes that "finding the movement within a stationary pose gives the figure energy that can be subtle or dynamic, depending on the pose and the artist's intention."

\section{Expression}

The human figure itself is an avenue for human expression. This is evident through the study of facial expression or gestural expression. Since gestural poses are action-oriented, they are often used to express some element of human sentiment in given environments and when this energy is combined with the profoundness of facial expression, the holistic human form becomes an indispensable tool for both expression and expressiveness.

\section{The Female Figure/the Male Figure}

The male and female forms are similar in structural composition but different in structural definition.
This is evident in the structure of both skeletons where pertinent parts of the skeleton are defined differently including the head, neck, shoulders, torso, arms, pelvis, legs and the length of bones and height of the entire skeletal frame. The female body is defined quite differently with a smaller head, long slender neck, shorter shoulders, slender arms, presence of waist and hips, slender legs and closer knees. The men have a much stronger frame with larger, more rectangular heads, short thick necks, wide shoulders, muscular arms and larger hands, thick torso and chest, muscular legs and knees that are wide apart.

\section{Individual Style}

Individual style is important in depicting how an artist chooses to render his or her drawings and how effectively that rendition is applied in the depiction of the intricacies of the human form. The use of a certain medium or a combination of various media culminates in unique effects that portray or highlight desired outcomes that the artist wishes to focus upon.

\section{ANALYSIS OF DRAWINGS}

\section{Body Shape and Gestures}
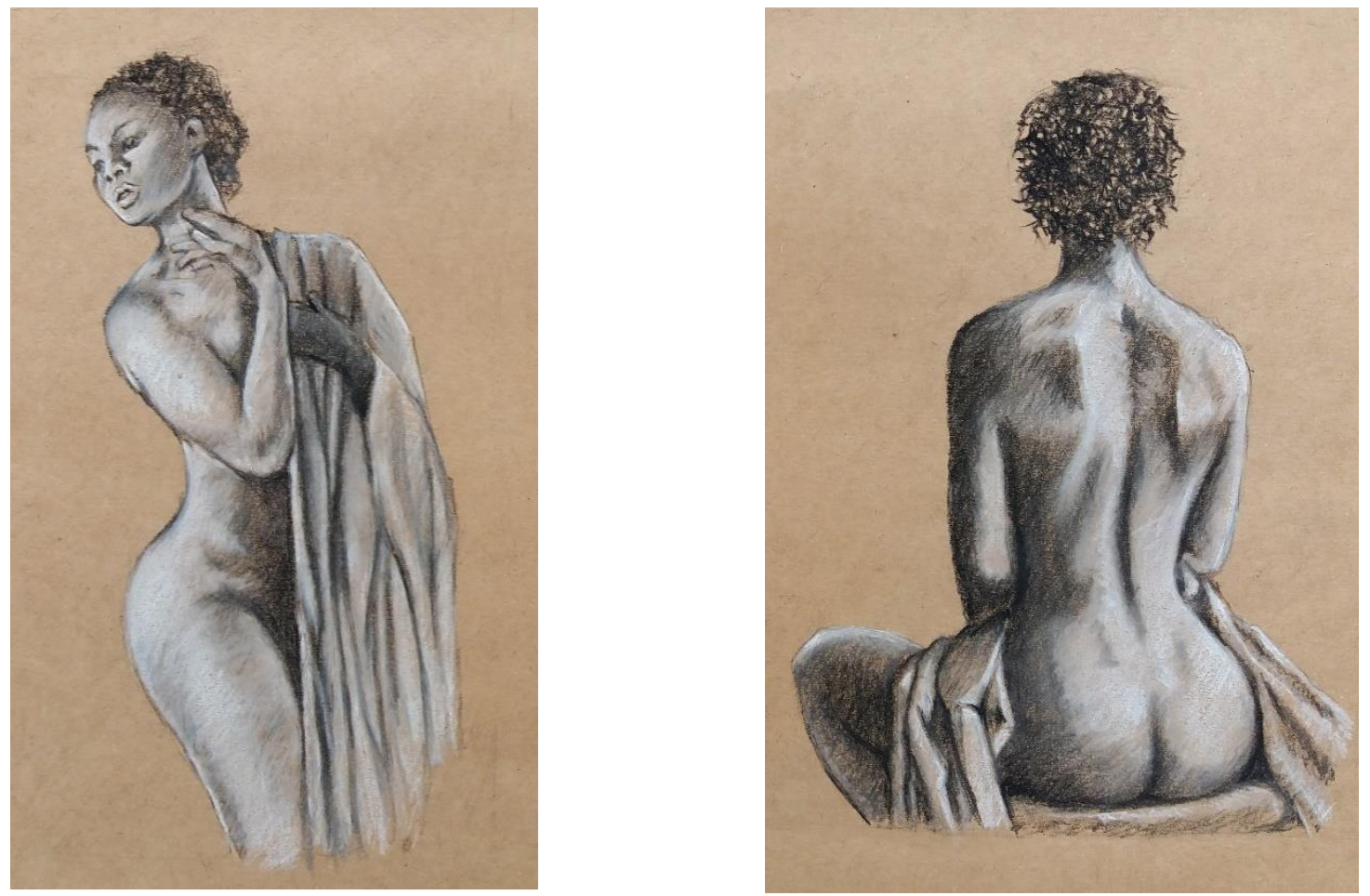


\section{Plate 1}

Value can be used to enhance lines, body shape, and gestures in human figure drawing. In Plate 1, the artist captures one half of the female figure, covering the rest in drapery. He uses tonal variation to depict parts of the body and captures a gestural pose that draws the attention of the viewer to the perceived beauty of the figure. Plate 2 features the female form viewed from the back capturing the proportions from the neck and shoulder axis to the placement of the back arms and the rest of the body curve, particularly the waist area. The artist uses tones to show the pertinent parts of the body viewed from this angle such as the bone structure and muscle formation on the upper back. It also provides him with the opportunity to study the upper body or torso which is important in human figure representation. He depicts the central axis of the torso or midline showing body symmetry when the figure is seated straight. The use of charcoal pencil is used effectively in creating a variety of dark and light tones as well as textural effects that culminate in the roundness of the form. The artist does not apply the smudge technique as would usually be the case and lets the textural effect of the pencil remain.

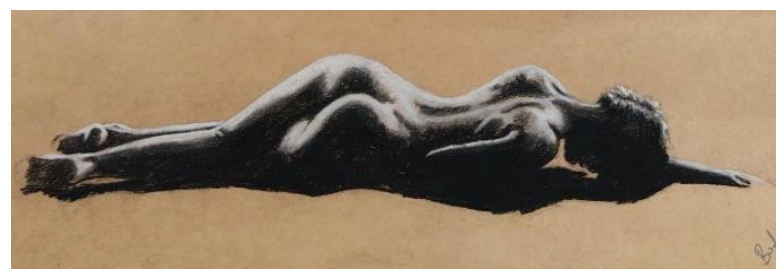

Plate 3

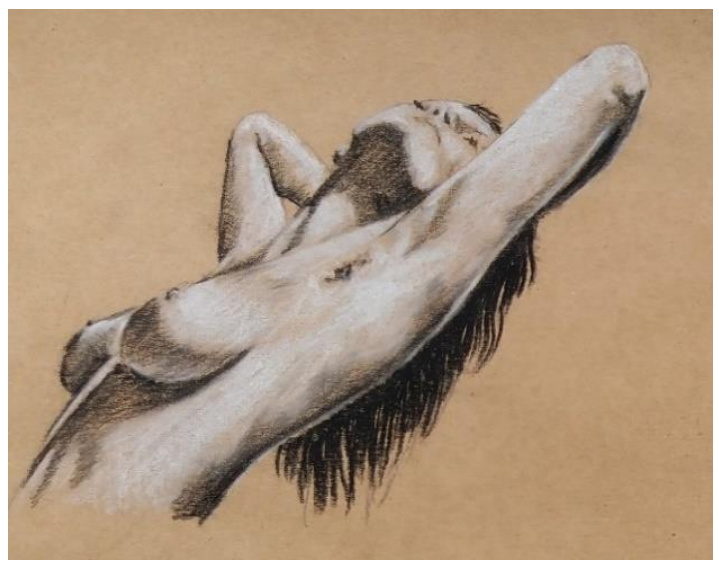

Plate 4
Plate 2

The use of light effects or the artistry of light falling upon certain areas, the use of dark, contrasting and dramatic tones that merge with shadows have often been used by artists to inject melodrama and sensuality to female human figure drawings. The artist uses this 'chiaroscuro' effect (Plate 3) to enhance the undulating contours of the figure using sharp contrast to emphasise pertinent parts of the body that attract the eye. He uses the effect of falling light to illuminate and underscore the parts of the body that the eye then easily picks out. This is done at an angle that carefully filters and controls the light in order to create the necessary contrasts and enhance the deliberate reclining pose. The shadows are used prominently and are merged with the edges of the figure such that there are no demarcation lines between them. This gives the figure instant grounding and a sense of sculptural intrigue. Such dramatic contrasts in female figure renditions are often used to demonstrate an element of sensuality and beauty of form beyond the normal configuration of the figure. The artist does not just want the viewer to merely see the accuracy of proportions but to feel the essence of the form's undulating beauty, or feminine body ideal, as placed in the environment.

In the following set of drawings, the artist seems inclined to show aspects of what can be described as the appeal of the feminine body.

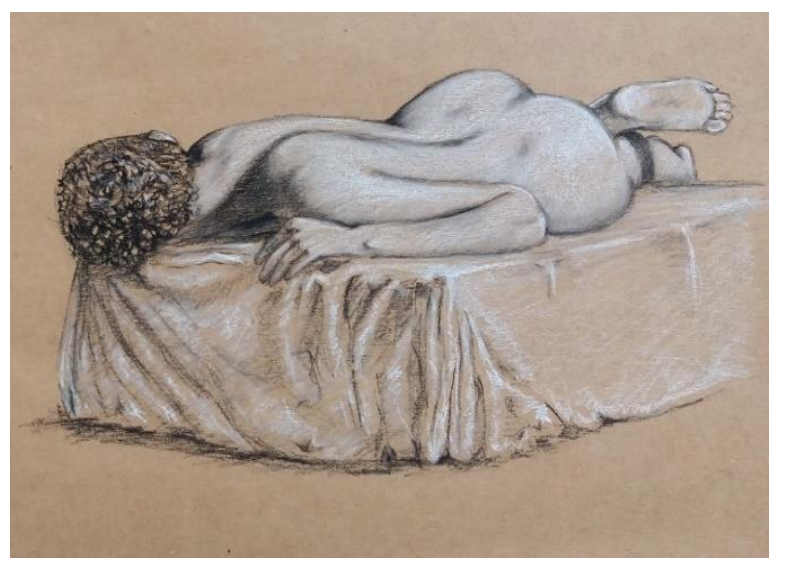

Plate 5 
Human figure drawing is used to study the suggestion of movement and the intricate nature of poses. In Plate 4, the artist captures what is called a 'stretch', a physically oriented pose that shows the elasticity of the female body when placed at a certain angle which necessitates an upward leaning stretch. This also suggests body motion and the physical artistry of the pose itself, including perspective. The suggestion of movement gives the figure a sense of vitality and meaning. The artist uses shading to show the roundness of the body and shadows to show protrusions such as the breasts and the head heavily tipping back revealing the underneath of the chin area. In Plate 5, the artist studies a pose that is both intricate and unique because of its angle of view. The foreshortening effect is applied on the legs and feet and defines the arrangement and fitting of the figure within the confined space of the pedestal. The pose demonstrates the pertinence of certain parts of the female body such as the protruding buttocks and how they manifest themselves when viewed from that angle. The artist captures the proportions of the body in a way that defines the figure despite the

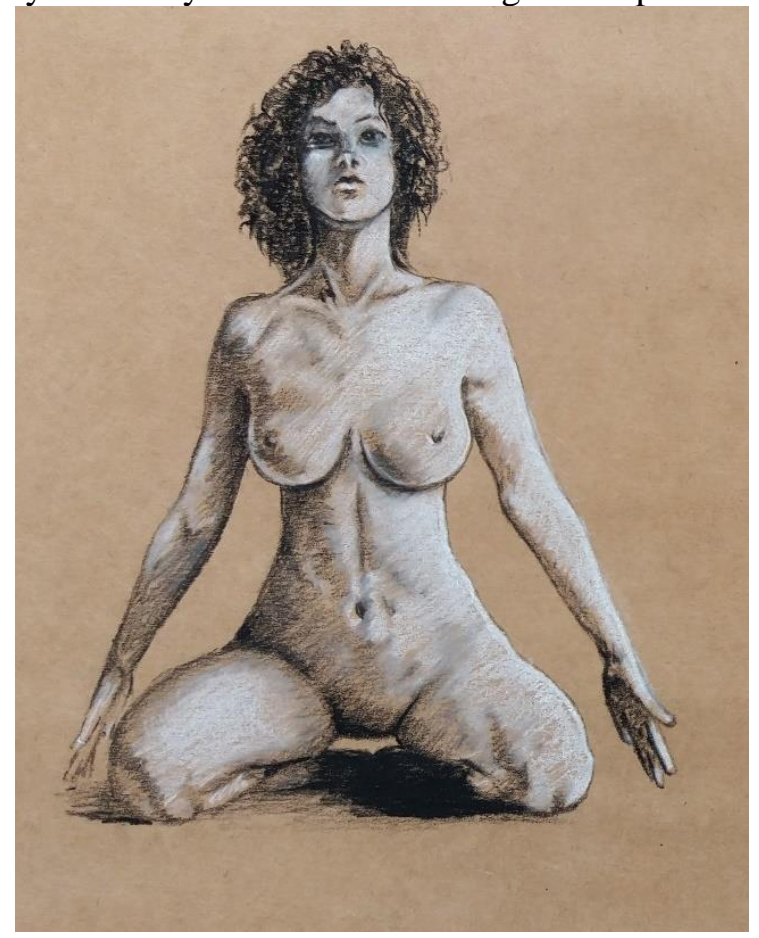

Plate 7

In the above drawings, the artist depicts the female figure in a way that creates a sense of curiosity that draws the viewer to the story of each woman. In Plate 7, the figure presentation is bold and direct, tricky angle of view and shows how the figure itself is flexible and manipulable.

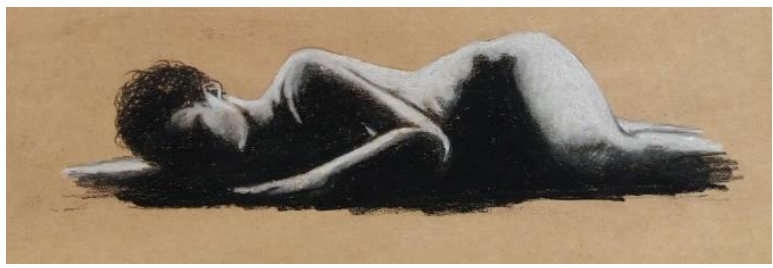

Plate 6

The artist revisits the use of chiaroscuro in rendering the figure drawing (Plate 6 ). The use of bold contrasts between dark and light areas as well as the emphasis of dark shadows adds visual weight to the reclining figure and creates a perception of threedimension. It can be said that the figure drawing presented this way, devoid of details and only illuminating certain parts of the figure from a controlled and deliberate source of light, creates a sense of mystery and drama. This is part of the creativity often applied by artists to raise the profile of the female nude form in order to delve into the possibilities of expressiveness that it bears.

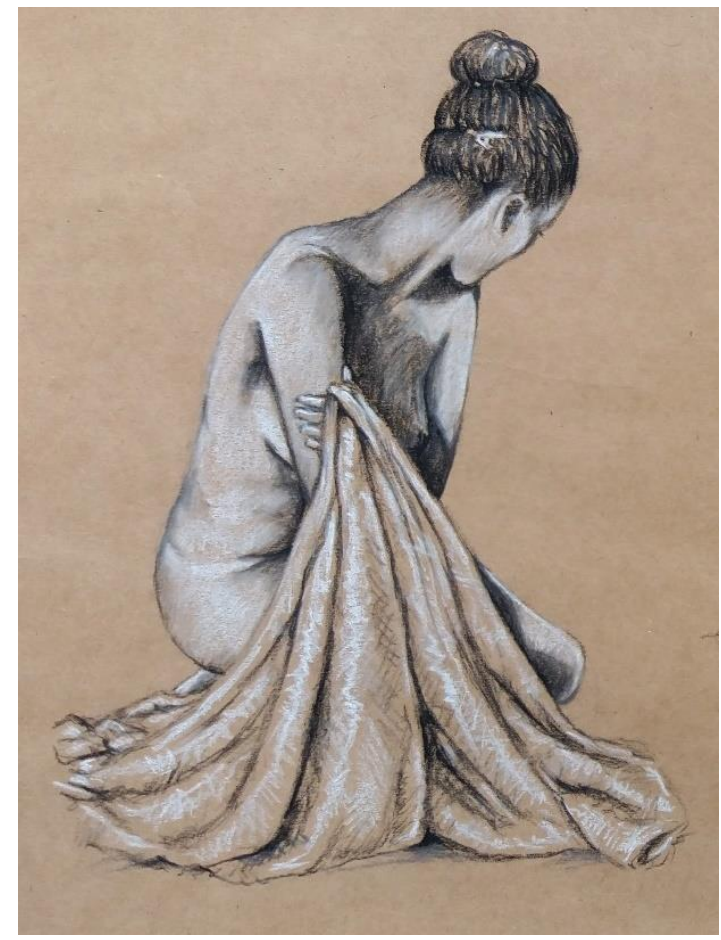

Plate 8

with the somewhat defiant pose signifying the power of the female form. Such studies often have nothing to do with depicting proportions as such, because that is already well-executed, but are a 
statement of something 'about' the female form. To emphasise the form, the artist uses light and dark areas as well as shadows to create a perception of three-dimension which makes the form rounded and captivating. The body proportions are, however, well captured and the study of the female anatomy itself is well articulated to become engaging and provocative. In Plate 8 , the artist depicts the woman covering herself with a draped cloth as part of the pose. Such poses are often designed to create a sense of curiosity and trigger imageries in the mind. The angle of view with the figure's face turned away

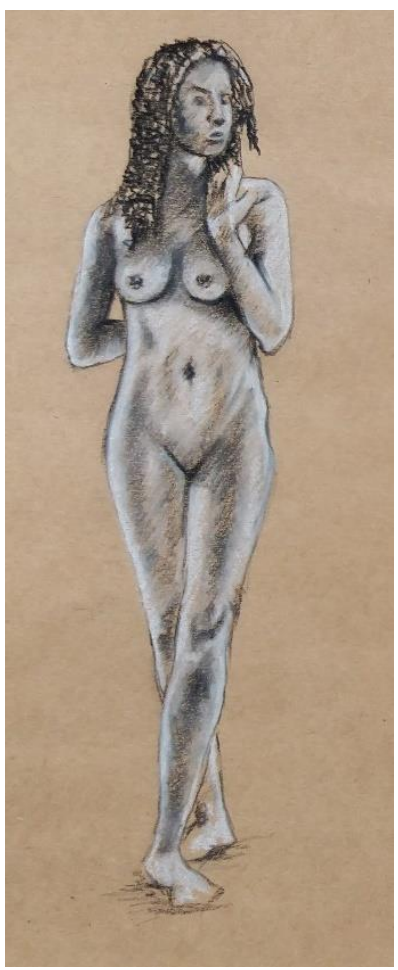

Plate $9 a$

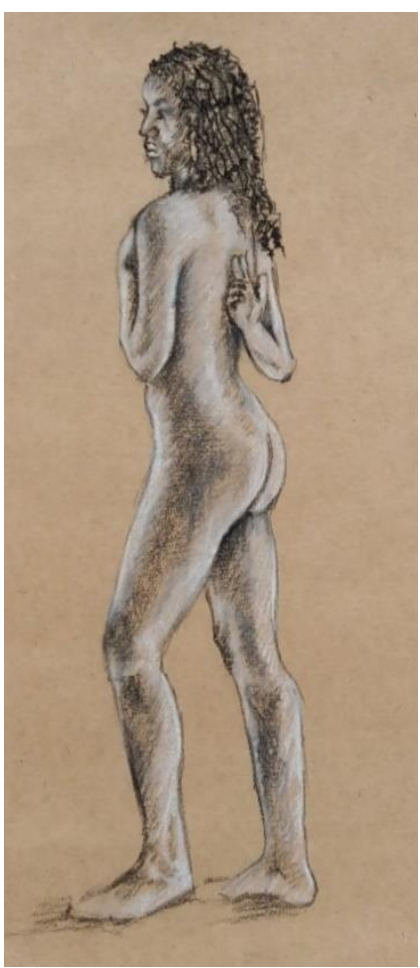

Plate $9 b$ from view is consistent with attracting attention to the figure through inquisitive curiosity. Whenever a face is turned away from the angle of direct view, there is always a curious need to turn it towards the angle of view to reveal itself. The artist takes time to study drapery, although, in the study of female nudes, heavy drapery is actually unnecessary unless the artist deliberately wishes to draw attention to some other aspect of the figure. The artist uses light and shadows to make the figure rounded and alive helping to suggest an aura of sensuality.
In Plate $9(a, b$ and $c)$, the artist studies a standing pose with a simple gesture from various angles to show the proportions of the female form as they appear from the front, side and back angles. The figures show the calibration of the female body from neck to toe. The artist uses the effect of charcoal pencil on toned paper to produce dark tones and the shining of light in certain areas to create the necessary contrasts that show the roundness of the body and its characteristics at any angle. 


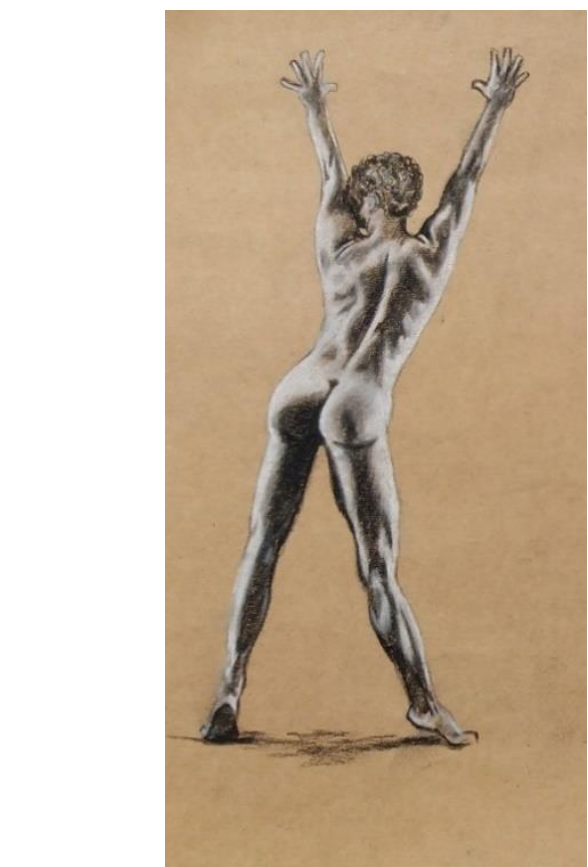

Plate $10 a$

With the intention of making the female human figure come alive, the artist injects a sense of energy, rhythm and movement into the next series of figure drawings. The dance-like movement (Plate 10a) is pleasant and energetic with light effects and dark tones used to emphasise body muscles and the tension in the limbs. The pose is created by carefully studying body structural alignment of the various axes, particularly the twist of the torso vertical axis and the lifting of the feet above the ankle line that denotes the action that necessitates the illusion of movement. Artists can

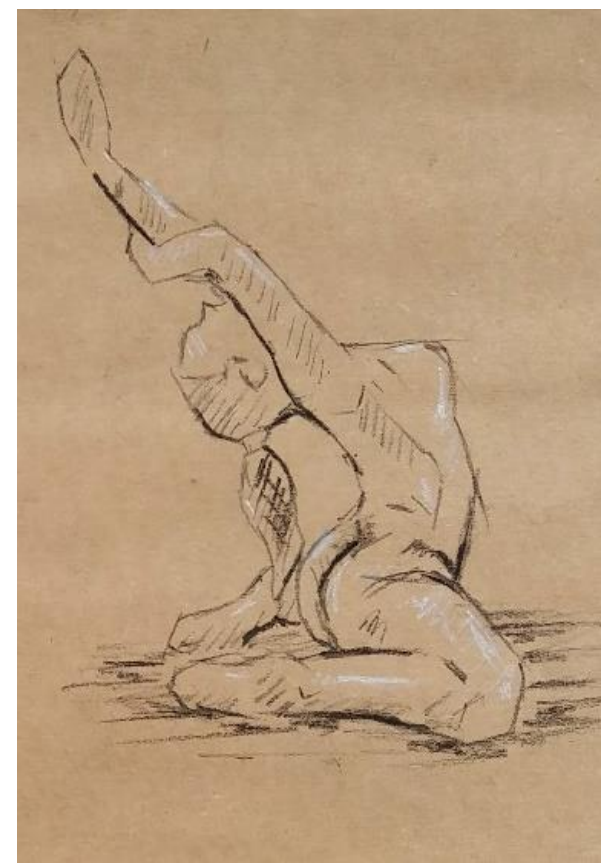

Plate $10 b$

use their imagination to create dynamism in a figure far beyond mere anatomical study or gestural representation. In making the studies the artist ensures that the figures bear good proportions enabling him to focus on enriching other aspects of the drawings. The uniqueness of this figure drawing is the deliberate use of light to illuminate certain parts of the body to enhance movement. The artist further demonstrates the flexibility of the human female form in a quick accompanying sketch (Plate $10 b$ ) that illustrates how a drawing can contain movement even though the drawing itself is still. 


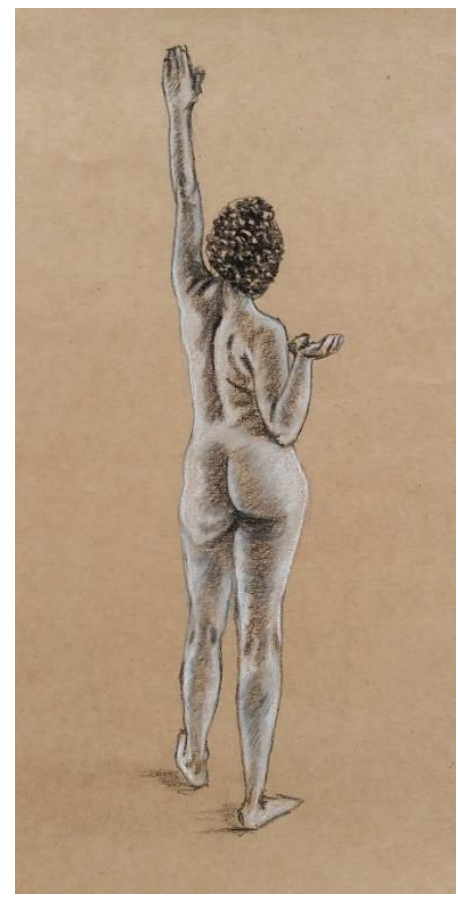

Plate 11a

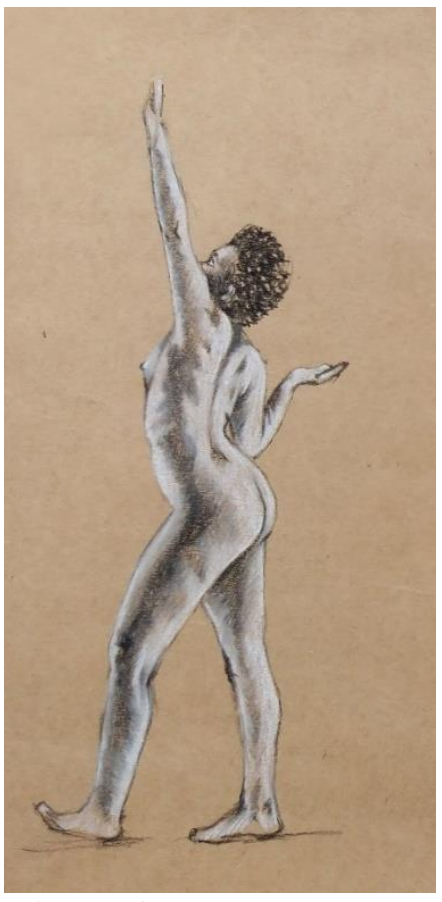

Plate $11 b$

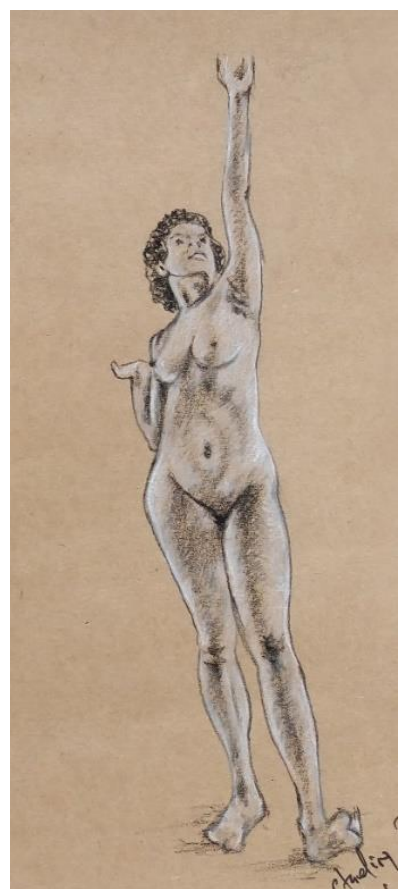

Plate 11c

In Plate 11(a-c), the artist creates a standing gesture study on a toned paper and proceeds to make it dynamic and energetic through the interesting twist of the body at the spine. This culminates in the pose bearing a specific action that makes it unique. He uses light and dark tones to create the illusion of three-dimension emphasising body roundness and body folds that underscore the movement (Plate 11a). This effect is enhanced by well-studied body proportions as well as the depiction of pertinent parts that ordinarily define the female form such as placement of buttocks, breasts and body curves.

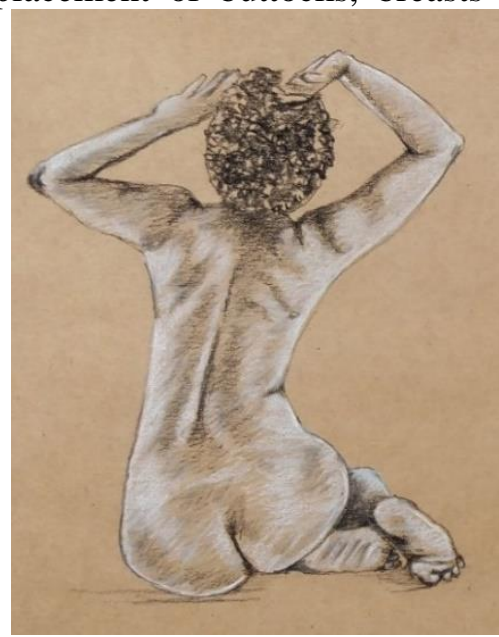

Plate $12 a$

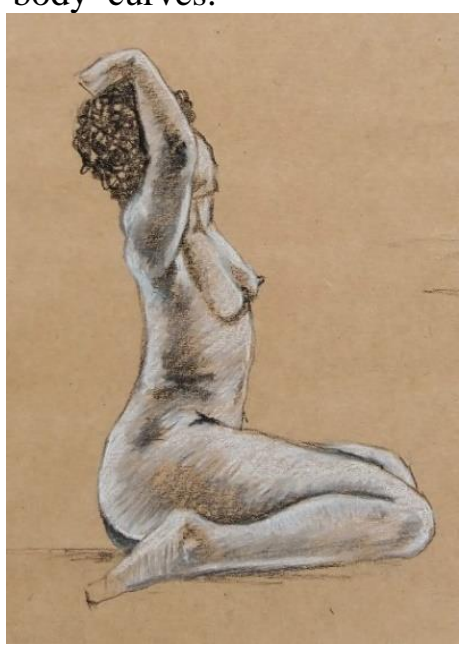

Plate $12 b$
Structurally, the artist references the horizontal and vertical axes of movement. In creating the pose, he exploits the flexibility of the central axis of the torso which he exaggerates a little to create a dynamic twist (Plate 11b). The lifted left-arm necessitates the steep angled raise of the shoulder axis to the left as well as the tipping of the hip axis also to the left. The pose also suggests the extension of the head and neck or tipping back of the head as well as the workings of the special antigravity muscles that ensure the standing body posture (Plate 11c).

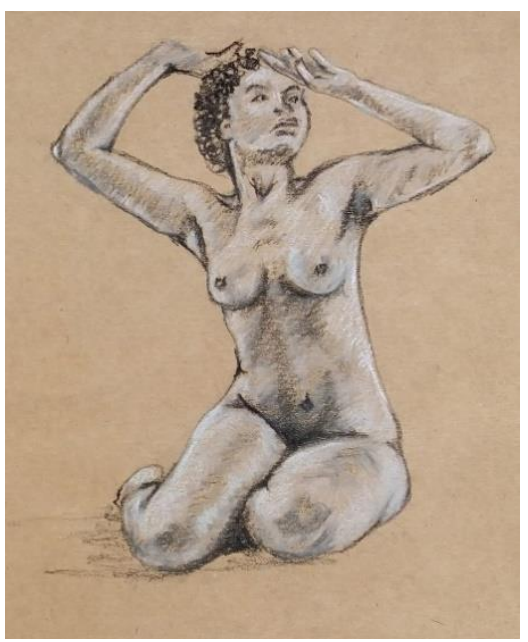

Plate $12 c$ 
In Plate $12(a-c)$, the artist studies a sitting pose with upraised/folded hands from various angles. As observed before, the creation of movement is based on the study of the mechanics of joints as well as that of the body's structural alignment. The drawings show how all parts of the body interlink and adjust, stretch, fold, and bend to create an action from all angles, yet retain their proportions. This means that despite the action pose, the figure retains its balance and beauty and does not become distorted.

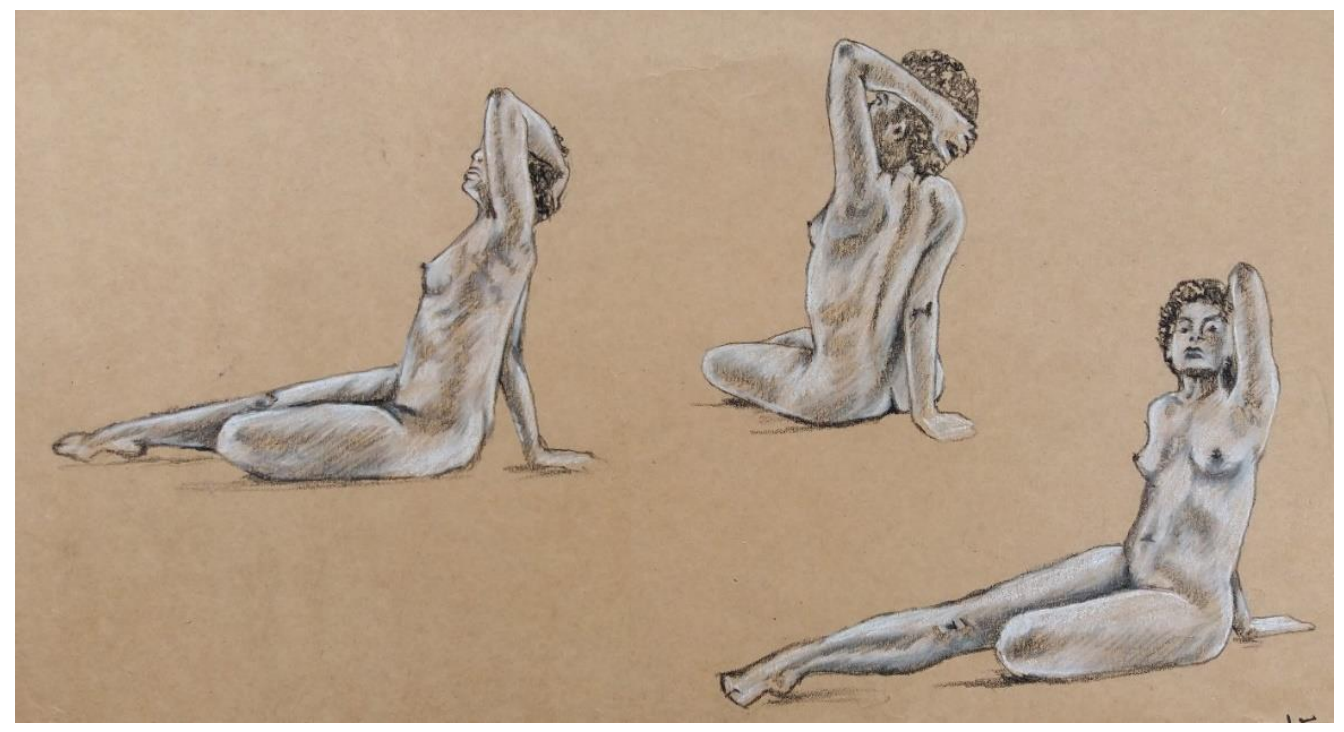

Plate 13

Plate 13 also features another action pose with one hand behind the head and the other anchoring the entire body frame creating a body stretch. The artist uses steady proportions to demonstrate how the different parts of the body manifest themselves in relation to each other, within the challenge of the pose. This study of the proportions in view of the

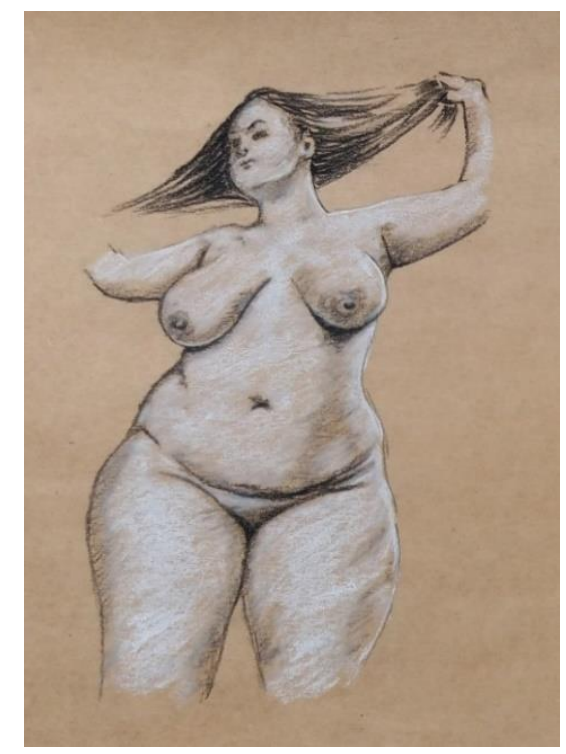

Plate $14 a$ particular pose also shows the flexibility of the body. The nature and extent of the body's twist or action is always seen in relation to the placement of the various axes and anatomical planes. The use of tones helps to make the body rounded and enhance the body's action.

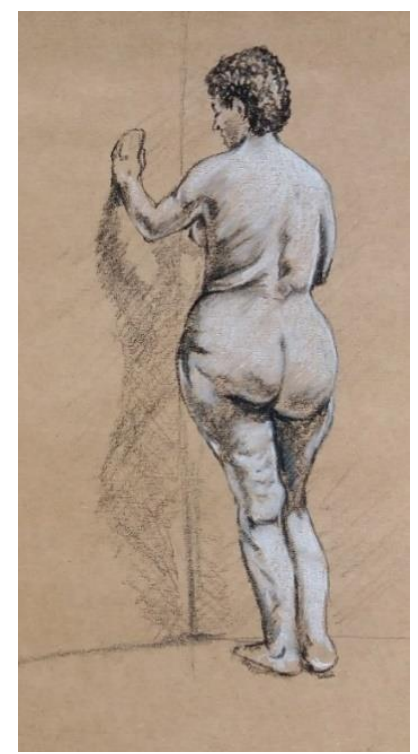

Plate $14 b$ 
Often artists focus and concentrate on what is termed as the 'ideal' female body or that which is described as possessing infinitely admirable proportions and physical beauty. But the study of a female nude also entails the study of the figure as it appears before an artist without bias of perceived 'artistic visual value' based itself on 'body judgment'. This body also bears its individual definition and characteristics. Subsequently, artists should not only tend to study the ideal but also study all realities, definitions and possibilities. The proportions of a female human figure remain constant irrespective of the physical description of the individual body and the task of the artist is to capture that description in its entirety and in its own uniqueness. The role of the artist is not only just to capture the figure, but to seek as well, to create an interpretation of the form, giving it energy, attitude and poise. The artist captures the essence of these two figures (Plate 14a and $b$ ) as a matter of routine study that underscores the notion of body positivity.

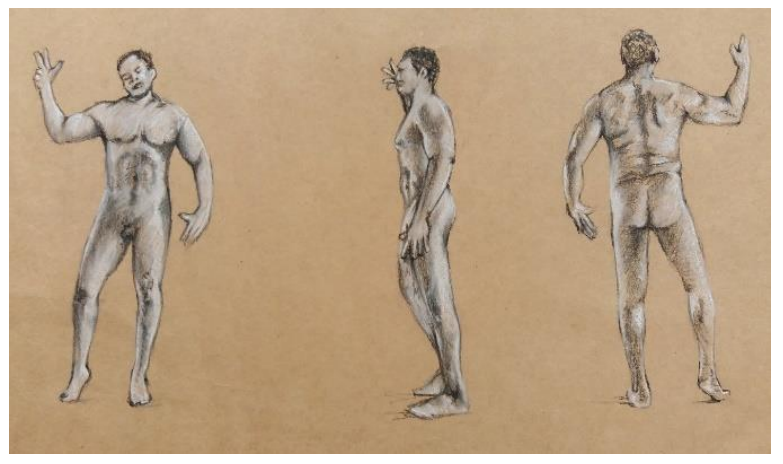

\section{Plate 15}

Plate 15 captures the body structure of the male figure in a simple gesture seen from three angles. It shows the study of proportions through the application of dark tones and falling light, a combination of which helps to identify and enhance the bone and muscle structure. As a result of the lifted right foot, the figure leans slightly to the left of the vertical axis with the body weight, therefore, shifted to the left side and resting on the left leg. This makes the torso and the swing of the arms slightly off-axis from the hips and legs (Contrapposto). The slightly angled neck helps to counterbalance the pose.

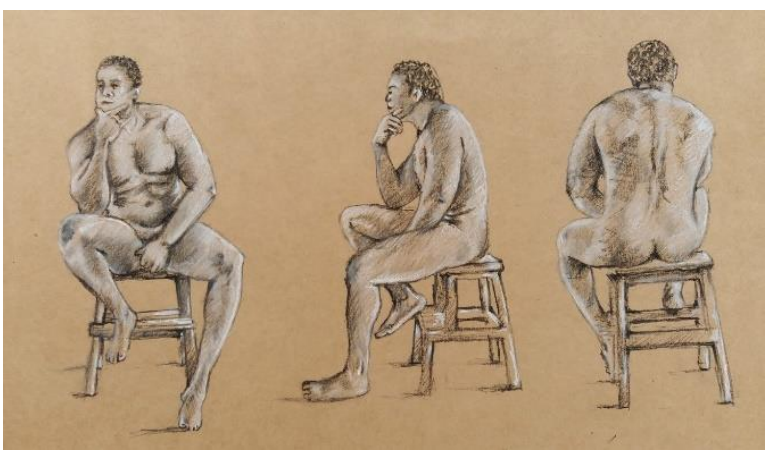

\section{Plate 16}

Plate 16 depicts a seated male figure from the three main angles. It shows how the proportions appear like in this pose and how they relate to the stool which is the support for the seated body frame. Since the elbow rests on the lifted right leg, it causes a slight shift of the torso to the right meaning that the body deviates to the right of the sagittal plane and bends the central axis of the torso to the right. The shoulder axis therefore falls to the right.

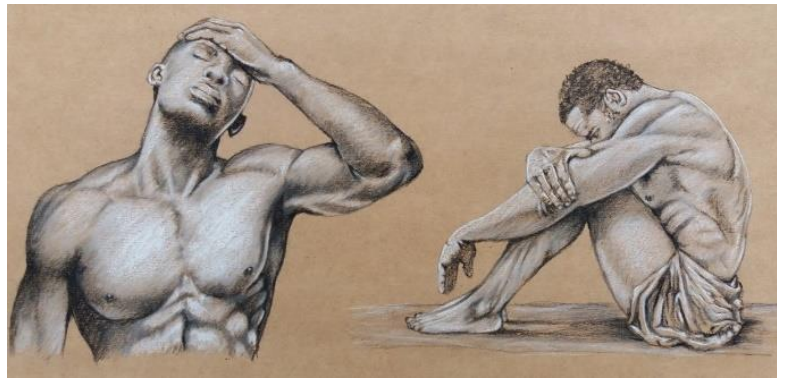

Plate 17

The anatomical study of the muscle structure of the neck, shoulder (deltoid, trapezius), chest (pectoral), arm (biceps) and abdomen is the subject of the $1^{\text {st }}$ drawing in Plate 17. The artist studies the muscles by the careful application of dark tones, light areas and shadows. It demonstrates the clear physical difference between the male and the female figure and propagates the usual narrative about masculinity and strength. In studying muscle structure ( $2^{\text {nd }}$ drawing), the artist references a sitting up pose with the body leaning forward with hands wrapped around the upraised knees and propped up leg. The proportions are drawn out relative to the pose and hence give an inclination of how muscles such as the triceps, deltoid, oblique abdominal muscles and other back muscles interlock to facilitate the pose. Often male human figure 
drawings are about studying the 'ideal' figure rather than capturing the regular proportions and most artists find this concept of the ideal quite captivating.

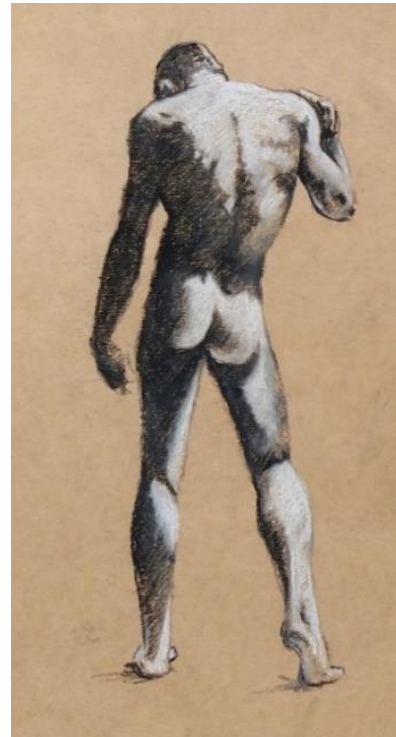

Plate $18 a$

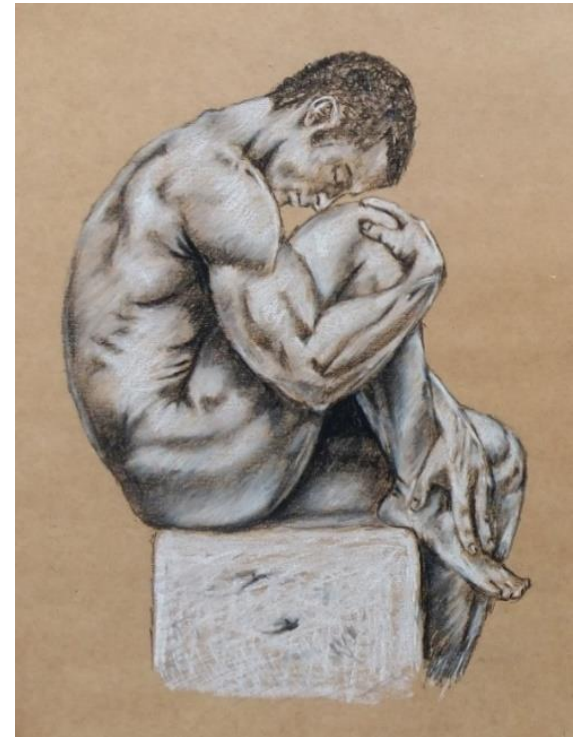

Plate $18 b$

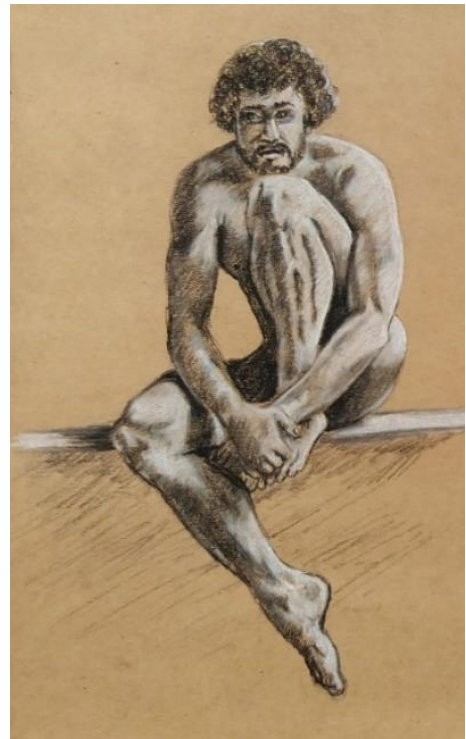

Plate $18 \mathrm{c}$
The artist studies anatomical details in each figure but lays greater emphasis on the muscular composition. In Plate $18 a$ the use of sharp contrasts and illumination focuses light on certain parts of the body that enhances the gesture of the pose and muscular contraction. The light illuminates the shoulder and back muscles down towards the pelvic pull, buttock muscles and rear thigh muscles as well as the calf muscles. This creates the feeling of threedimension on the contrapposto pose and makes the figure dramatic. Plate $18 b$ and $18 c$ are both studies of intriguing muscular composition and the poses are designed to bring out the intricacy of muscle interrelationships and mechanics that constitute the body functionality of the male figure as contained in the anatomical study of shoulder, back, arm and leg muscles. The poses are not just regular gestural poses or specific action poses but are deliberate compositional poses where the body is 'arranged' to create maximum display of musculoskeletal and anatomical possibilities.

\section{The Hands}

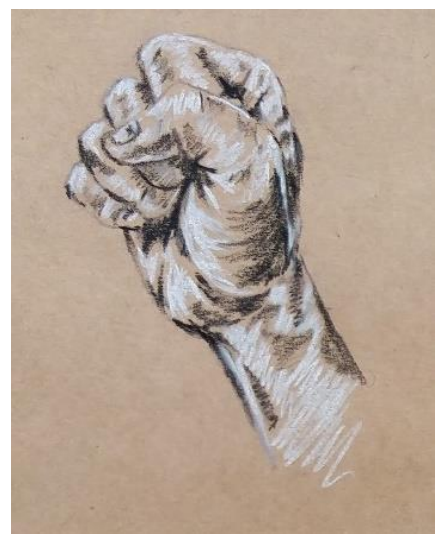

Plate 19a

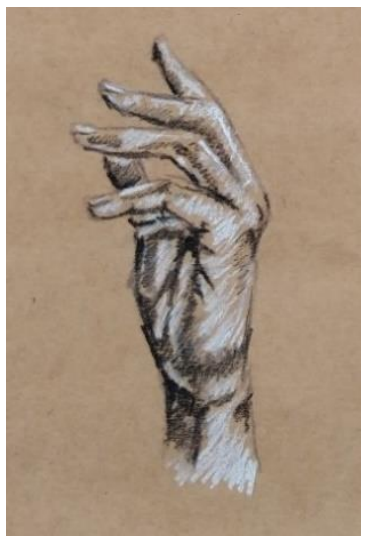

Plate $19 b$

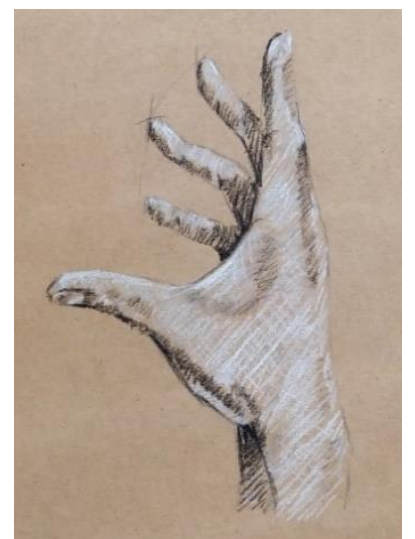

Plate $19 c$

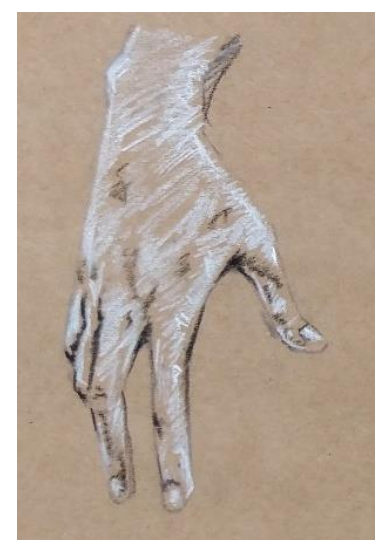

Plate 19d 
In Plate $19(a-d)$ the artist makes gesture tonal studies of hands. Hands are complex and are not easy to draw but provide a good opportunity to study the entire complexity of the human figure. Hands are an integral part of human figure drawing since they form an important part of the body's functional ability and therefore its eventual calibration of proportions. A human figure drawing, whether male or female cannot be visually complete without the inclusion of hands and feet in their correct proportions. Hands are also extremely important in the formulation, creation and depiction of gestures and action, which form the core element of human expression. Indeed, without the presence of hands and feet, the rest of the body itself would be completely devoid of expression and character even with the presence of the face. In order to focus on the composition of the hands themselves, it is always advisable to isolate them and study them on their own. The artist makes straightforward drawings depicting various gestures, studying the wrist, finger segments and the knuckles which are the joint areas. Plate $19 a$ is a strong grip while the rest (Plate 19b-d) are various poses of open hands featuring dorsal/ventral angles. He then applies various light and dark tones to create a threedimensional effect which helps to show how fingers break into hand movements.

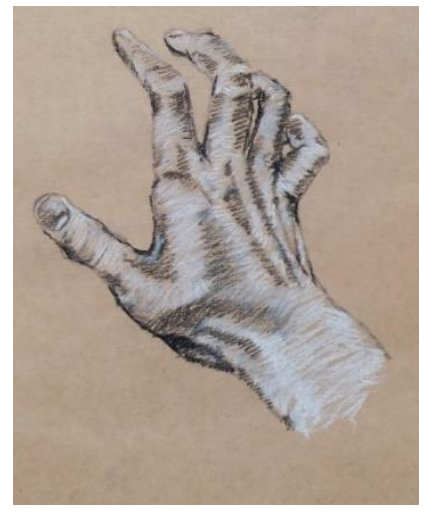

Plate 20a

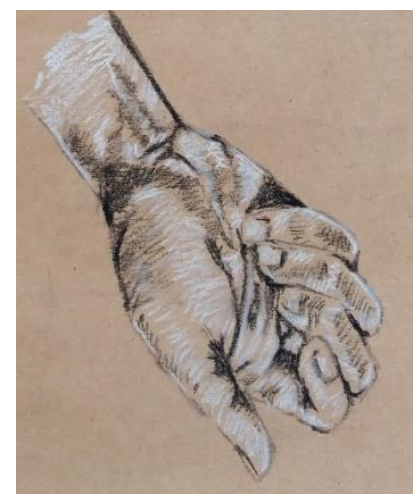

Plate $20 b$

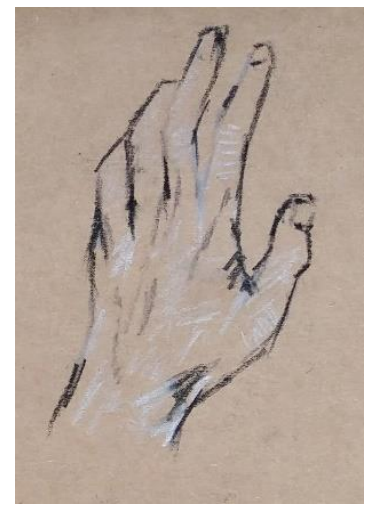

Plate 20c
Plate $20 a$ shows the study of the finger segments folded at the joints and the pull of connective extensor tendons which creates a reach-out hand grip pose which is associated with a certain expression akin to fear. Plate $20 \mathrm{~b}$ depicts the study of the grasping effect of the ventral hand and Plate $20 c$ is a regular partially outstretched dorsal aspect of the hand. It is important for an artist to be aware of the musculoskeletal anatomy of the hand and factor it when drawing parts of the hand because ultimately the hand is a functional item and drawings must depict this functionality just as much as they depict the artistic rendition.

\section{The Feet}

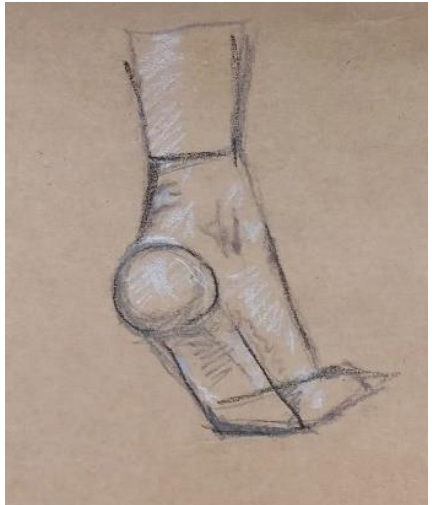

Plate 21a

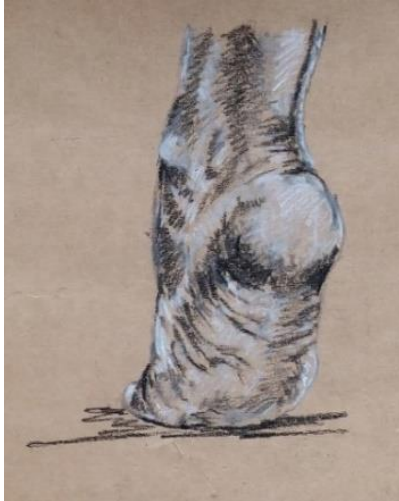

Plate $21 b$

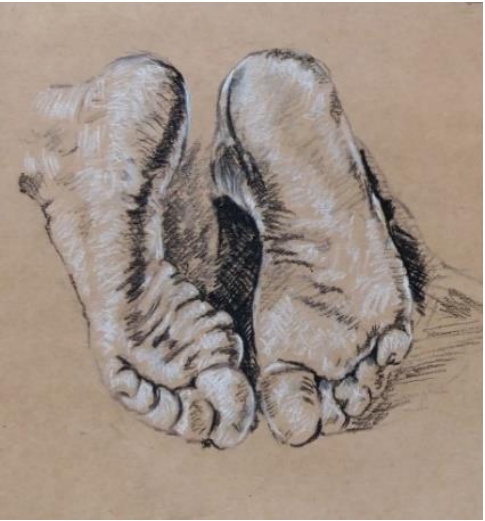

Plate 21c 
Feet are always anatomically used as the base balance of the entire body structure and are aligned with the special antigravity muscles that aid the standing body posture and facilitate grip and mobility. They are, therefore, an integral part of human figure drawing because they not only visually complete the figure but also contain the structural mechanism through muscles, tendons and ligaments that make the entire body have its posture, ensure mobility and facilitate gestures and poses. The artist makes a quick study of the heel bone which he captures as a circle and connects it to the standing metatarsal bone through a rectangular suggestion of the arch, forming the raised foot (Plate 21a). He makes a slightly more detailed study of the same on the right foot showing how the weight is distributed all the way down to the tip of the sole (Plate 21b). He then makes a study of the bottom of feet showing the heels, the fat pads, toes and the big toe, as well as the inner and outer longitudinal arches (Plate 21c).

\section{OBSERVATIONS AND OVERVIEW}

In reference to the study of the female figure, it is observable that the artist, in referencing photographs, creates his figure drawings from inspirations derived from several perspectives.

\section{Study of the Female Body as a Unique Creation}

The artist creates his drawings depicting the female figure as a unique and beautiful creation in its own right. In this regard, he takes time to study the proportions of the body that culminate in the presentation of this uniqueness and beauty. The studies in Plates 9 ( $a, b$ and $c$ ) are straightforward depictions of the female figure from different angles that show the physical body and its proportions. Though the figure displays a gestural pose, the artist does not seem to make any statement other than to show how the female figure is calibrated; from the head to the narrow shoulders, the bosom and breasts, the torso, the pelvic area, the hips, the thighs, knee, lower leg down to the ankles and feet (Plate 9a). He proceeds to give side and back view that show the formation and shape of the torso, shape of the arms and hands, the slender waist area, protrusion of the buttocks as well as the shape of the legs and the stability created by the feet. In terms of anatomical presentation, he therefore suggests the formation of the bone structure and the various joints that constitute the human female frame (Plates $9 b$ and $c$ ). These drawings portray the elegance and delicateness of the female figure as a basic study.

\section{Movement and Gracefulness}

In other studies, artists ventures beyond the simple figure and injects movement and gracefulness to the figure drawings. The artists study the beauty of movement that emanates from the body making it alive and graceful. In the series of studies, the artists demonstrate that the human body and particularly the female body, is not static and by its composition bears infinite elegance. It can be stretched and modelled, constricted and folded; it can be made to respond to musical inclinations, like ballet or the intricacies of Yoga. (Plate 10a \& $b$ ). Other drawings that depict the elegance of movement are also gestural poses that show the stretch of the body in various angles that also show how the body twists in tandem with the functional role of every part of the body and joints (Plates 11a-c). Since the body is not static, movement becomes an important part of its description and expressive possibilities.

\section{Use of Nudity as a Way of Expressing Feminine Beauty}

In studying the movement of the body, the artist also delves into displaying the female nude body as a tool for the expression of feminine beauty. In Plate $12(a-c)$ and Plate 13, it is doubtful whether the artist is simply studying seated gestural poses from various angles. It shows a deliberate effort to showcase how the nude body possesses expressive potential, almost an invitation to its own beauty and poise. In Plate $12 a$ the body has a slight twist to the right showing body curves and the placement of the buttocks next to foreshortened legs and feet. The effect of nudity is to showcase the body as it naturally manifests when placed in such an environment. Plate $12 b$ shows the same body from a side view that depicts the stretch of the full thigh and the folded leg as well as the slim upper body. Plate $12 c$ shows the front view with the full display of the placement of the stiff breasts as an item of artistic study as well as the fullness of the thighs and poise of the body. The artist continues with this line of study in Plate 13. It cannot be denied that the 
study of this gestural pose was not only for purposes of artistic aggrandisement but had perhaps more to do with the possibilities associated with the physical attractiveness that the female body exudes and its suggestion of sensuality. In depicting the power of femininity, the artist creates two interesting drawings; one that is bold and the other suggestive of sensuality. In Plate 7 the drawing, by virtue of its gestural pose, is a bold 'presentation of self'. It is not about the study of proportions; the artist has managed those quite well already; it is about a feminine statement.

\section{Suggestiveness and Youthful Femininity}

In some of the drawings, the artist studies the female form but introduces attire that covers parts of the body. Such drawings create a sense of visual suggestiveness since the drawings leave certain pertinent parts to the viewer's imagination. By so doing the artist draws the viewer's eyes to the clothed areas as if to unveil them and complete the nudity, since the eyes always seek a sense of completeness. This effect is particularly evident in Plate 1 and Plate 8.

\section{Romanticising the Form}

It is observed that in some of the drawings, the artist suggests an element of romanticising the female form through the application of a specific style of shading. The chiaroscuro effect or the use of sharp contrasts between light and dark areas as well as the application of shadows, illuminates certain parts of the female form, creating a feeling of mystery and power. This is far removed from the normal study of the proportions of the form and seeks instead to deliberately explore the allure of the form's hidden meanings. In Plate 3, there is no other reason for the figure to be reclining face down other than to facilitate the illumination of certain parts of the upper body and create shadows, underscoring not only an illusion of depth but also a sense of the perfect undulating body, idealism and mood. One of the most important reasons for the use of the chiaroscuro effect is to draw the focus of the eye towards certain pertinent parts of the figure that the artist wishes the viewers to observe. In noting the effect of the use of chiaroscuro, Scott (2019) observes that "our eyes are naturally drawn to the lightest areas, so these tend to be the focal points in the painting." In Plate 6, the artist does not include many details but lets the eye capture the lying form through illumination and the application of shadow effects.

\section{Body Positivity}

The two drawings that are different from the others represent a different thinking that is admirable. As observed before, artists love idealism; the perfect almost unreal form, made of minute waists and woefully lean bodies. But there is also the 'real', not the ideal, which cannot be ignored or wished away. The artist sees this as a 'type' of body that bears its own characteristics and needs to be celebrated as it presents itself. This is generally associated with body positive art and the wider notion of body positivity. "Body positivity refers to the assertion that all people deserve to have a positive body image, regardless of how society and popular culture view ideal shape, size, and appearance" (Cherry, 2020).

\section{CONCLUSION}

Human figure drawing remains fundamental to the creation of all artwork. This is because artists and other creative people view the human figure, be it male or female, as an embodiment of the 'self'. Subsequently, they create it in their own image, as a mirror reflection of their physicality and their spiritual existence. The human figure is, therefore, inextricable from the realm of human expression. Artists then draw and study the human figure in order to acquire and develop the necessary all-round skills that help them to best use it as an expressive tool in whichever discipline or genre of art that they choose.

In figure drawing, the choice and purposeful use of medium or a combination of media is important in rendering the effect that an artist wishes to render in order to meet his or her visual-expressive objective. This choice is also important in executing the technique suitable for this objective. The choice of medium and the rendering technique or indeed the wider personal style are all intertwined and work methodically to construct the drawing. This culminates in individual touch that to a large extent, defines the quality and effectiveness of the work which ultimately sets it apart. 
Human figure rendering can be used to express certain kinds of feelings and emotions making them a conduit for artistic expression by the individual artist. Every time an artist wishes to create a piece of art, the major consideration is often the placement and role of human figures in his/her composition. Whether in drawing itself, painting, or sculpture, these human figures, placed strategically within the picture plane or as an integral part of the sculptural form, become the focal point of expressional intent of artist. They are, of course, presented together with other compositional elements and principles but they become the beacons of expression through facial expressions, gestural actions, exaggerations and suggestion of movement. All these are used to delve into the intricacies of human feelings, emotions and moods as espoused by the artist.

Human figure drawings, depending on how they are executed and particularly in combination with facial expression, can also be used to elicit certain types of emotions from the viewers, meaning that they can bear expressiveness. In paying particular attention to the viewer, the artist can present the human figure in a way that the figure engenders a mood, emotion, or a 'suggestiveness' that is not necessarily a direct emanation of the artist's own feeling or personal expression. In this regard, certain human figure renditions become bold communicative or propagative tools of given social or human phenomena. It must be understood, though, that all expression ultimately emanates from the intricacies and complexity of the human spirit. The point here is that on one hand this expression seen through figures is derived directly from the individuality of the artist, or it is on the other hand, elicited from the viewer by virtue of the contextual undertones or implications of the figure representations.

Gestures, through their action and the movement they suggest, are core to the study of the human figure. The essence of the study of the human figure itself is not just the satisfaction of getting its configuration right, but the expression it underscores and the freedom and possibilities it provides the artist. Hence gestures, through their ability to direct the eyes, are important for all types of expression of human feelings, emotions and moods. When the action gesture is removed from the human figure, it leaves the artist and the viewer alike, with a static outline that amounts to no visual meaning.

\section{REFERENCES}

Cherry, K. (2020). What is body positivity? Retrieved on December 21, 2020 from Verywellmind.com, https://www.verywellmind.com/what-is-bodypositivity-4773402

Errington, W. (2015). The Female body in Art as a Non-Sexualised Being. Retrieved on December 19, 2020 from The-artifice.com, https://theartifice.com/female-body-art-non-sexualisedbeing/

Hutter, H. R. (2020). Drawing art'. Retrieved on December 27, 2020 from Britannica.com, https://www.britannica.com/art/drawing-art

Pelissier, S. (2018). About women representation in Art. Retrieved on December 22, 2020 from Paintingdemos.com, https://paintingdemos.com/ about-women-representation-in-art/

Robinson, J. (2007). Expression and expressiveness in Art. Postgraduate Journal of Aesthetics, 4(2), 19-41

Scott, D. (2019). Chiaroscuro. Retrieved on December 21, 2020 from Drawpaintaacademy.o rg, https://drawpaintacademy.com/chiaroscuro/

Walter, E. (2019). The evolution of the female body in art. Retrieved on December 19, 2020 from Theboar.org, https://theboar.org/2019/04/evolut ion-female-body-art/

Winslow, V. (2008). Classic Human Anatomy in Motion: The Artist's Guide to the Dynamics of Figure Drawing. Retrieved on December 23, 2020 from Doctorlib.info, https://doctorlib.info/ anatomy/classic-human-anatomy-motion/12.ht $\mathrm{ml}$

Photograph Reference Sites:

http://www.female-anatomy-for-artist.com/

http://www.human-anatomy-for-artist.com/ 